\title{
Conceptual exploration of a gravity-assisted electrorheological fluid-based gripping methodology for assistive technology
}

\author{
Ivo Radice ${ }^{1}$ • Jeroen H. M. Bergmann ${ }^{1}$ (D)
}

Received: 13 June 2019 / Accepted: 7 August 2019 / Published online: 31 August 2019

(c) The Author(s) 2019

\begin{abstract}
Gripping devices help patients carry out everyday tasks and increase their independence. However, there seems to be a lack of bionic gripping technologies that can fully adapt to any possible shape, as the use of artificial fingers and predetermined grip settings limits the operating space. The development of a more agile device, which is operated by a simple control paradigm, could greatly benefit users. An electrorheological (ER) fluid system should be able to adapt to the shape of an object and then hold that configuration. The aim of this study was to explore if a conceptual prototype of an ER system could hold a geometric shape when it is activated. A test rig was constructed with a moving part (set in different silicone oils) that could be displaced using a tensometer. Silica particles were dispersed in the silicone oils, and a field with a voltage of $4 \mathrm{kV} \mathrm{mm}^{-1}$ was generated to activate the fluid. The results show that the developed system can support an increased force when activated and hold a simple geometric position without any noticeable delay. This outcome provides an initial proof of concept for a possible new (gravity-assisted) gripping approach using smart fluids, which could be developed with materials that are biocompatible and widely available.
\end{abstract}

Keywords Smart materials $\cdot$ Prosthetics $\cdot$ Exoskeleton $\cdot$ Grasping $\cdot$ Manipulation $\cdot$ Assistive technologies

\section{Introduction}

The change to bipedalism and the development of truly dexterous upper limbs can be thought of as key physical evolutionary markers that started mankind's control of our planet. The greater part of our interactions with our environment is via gripping actions. This reliance on the hands means that even a slight change in hand capabilities leads to a change in behavior [1]. There are many devices described, which aim to replicate hand function [2-4], and most of these technologies tend to replace the movement of muscles, with artificial tendons or actuators. Some proposed systems take a

Electronic supplementary material The online version of this article (https://doi.org/10.1007/s42242-019-00048-5) contains

supplementary material, which is available to authorized users.

\footnotetext{
Jeroen H. M. Bergmann

jeroen.bergmann@eng.ox.ac.uk

Ivo Radice

radicei@stedwardsoxford.org

1 Department of Engineering Science, Old Road Campus Research Building, Institute of Biomedical Engineering, University of Oxford, Headington, Oxford, UK
}

different tactic and use for example shape memory alloy actuators to selectively change the stiffness of the finger joints [5]. In general, contact pressure or geometric constraints can prevent an object from slipping while it is being gripped, and these are the two fundamental ways of holding on to objects. The complexity of the grip control is dependent on the selected technique and design, with geometric constraints providing a more elegant and simple solution.

A robotic gripper that uses the principle of jamming of granular material successfully applies the idea of geometric constraints to pick up a range of objects [6]. Developing the robotic gripper into a more mobile and appropriate version for patients is challenging. Ideally, the benefits of a geometric gripper would be combined with user requirements, such as a fast response time, low power consumption and silent operating mode for everyday use as well as the ability to have the system battery-powered, so it can be made wearable. Electrorheological (ER) fluids, which change their rheological properties (viscosity and viscoelasticity) under the influence of an electrical field [7], are a promising candidate for such a system. These smart fluids are applicable in widely used mechanical devices, such as clutches and brakes, and they can change their shear stress almost instantly [8]. ER flu- 
ids have the benefit of reversible continuous control and fast responses, which also make them ideal for vibration control, tactile displays and sensors [9]. These features give us the opportunity to develop a new range of devices and concepts for the biomedical field. ER spherical joints and pistons have already been proposed to aid minimally invasive surgery by optimizing haptic control [10]. Furthermore, magnetorheological fluid brakes have been suggested for a haptic gripper [11]. This shows the potential that smart materials can have upon the field of assistive technologies.

However, these methods have not yet been adopted to explore more dynamic gripping methodologies. Smart ER fluids can meet the design requirements of a gripping system, while benefiting from the ability to quickly transition between an "unlocked" and "locked" state by responding to an electric field in the order of milliseconds [12, 13]. A simple and elegant gripping approach could rely on gravity to position the "fingers." The fingers can consist of a movable electrode within the ER fluid, and they can be "locked" by the application of an electrical field. Such a system can also fulfill the basic requirements of a grasping device for assisting humans in their daily lives. This is especially true for picking up objects at ground level, as the orientation of the gripper will allow for the gravity assist that drives the fingers around the object.

The effect of a high-voltage electrical field on fluids composed of particles suspended in a dielectric liquid was discovered accidentally by Winslow [14]. He observed that "there is a tendency for the suspended particles... To form an infinite number of strings or lines extending between the [electrode] plates while under the influence of potential." $\mathrm{He}$ then noted that these lines disappear as soon as the circuit is broken. He attributed the greatly changed properties of the fluid in the electrical field to these lines.

Three decades later, JE Stangroom describes the minimum requirements for a fluid to show an electrorheological response [12]. The base liquid must be hydrophobic; the suspended particles must be hydrophilic and porous and must have absorbed water. He explains why this water is needed and how the lines that Winslow initially observed are formed. It was assumed that there are mobile ions present in the pores of the solid particles. "When an electric field is applied to the fluid the ions move to one end of the particle, carrying the water with them. One end of the particle therefore becomes overtly wet and water 'bridges' can form." These bridges join to each other to create the chains that result in the observed property changes [12]. This is now known as the water bridge theory (see Fig. 1), and while other theories have been proposed to explain exceptions, the water bridge theory is now widely accepted and provides a good working model to explain the emerging properties when an electric field is applied.

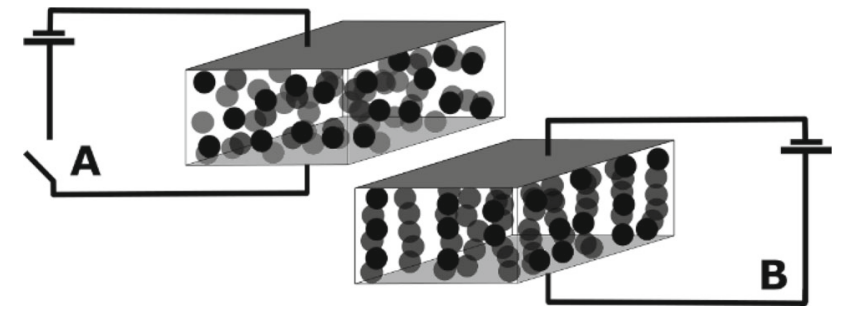

Fig. 1 ER fluid between two high-voltage plates during a zero-voltage condition (a) and a high-voltage condition (b). The images show the change in structure that occurs under the influence of a high voltage potential. The chains that are formed can be explained by the water bridge theory. The spheres represent silica particles dispersed in silicone oil that are situated between two electrode plates. The schematic circuit further consists of a DC voltage source and a switch that is open in (a) and closed in (b)

A review on the contemporary understanding of ER fluids described the ways in which ER fluids are typically tested and how shear stress is used as the dependent variable [15]. The review goes on to describe and quantify the power law relationships that the electric field, composition and temperature have with shear stress. It confirms Stangroom's assertion that the current does not follow Ohm's law. It suggests that the power varies depending on the fluid and the field strength. The experiments show in detail the formation of structures in ER fluids. The data imply that below $0.5 \mathrm{kV} \mathrm{mm}^{-1}$ no complete chains form between electrodes, though some accumulation along field lines is noted in smaller fields. The mechanisms by which the ER effect works agree with the explanation of the water bridge theory and lay out a theory that explains the effect of using the polarization of particles. This polarization theory accounts for how an ER effect has been observed in fluids that are anhydrous. This alternative theory does not discount the water bridge theory, but rather explains how different mechanisms can produce the same end result $[12,15]$.

The response of the ER fluid system itself also differs substantially between alternating current (AC) and direct current (DC) fields, but the time of response is similar for both these fields [16]. The response time decreases, as the field strength increases, and the results suggest a nonlinear dependence between DC field strength and dynamic yield stress that mirrors the previous findings [15].

A study that compared responses of four laboratoryprepared ER fluids and one commercial fluid observed that the response times varied with field strength, field type (AC/DC) and the base viscosity of the liquid in which the silica particles were dispersed [13]. These results on varying field strength confirmed that the ER effect only seems to take place at strengths above $0.5 \mathrm{kV} \mathrm{mm}^{-1}$ [15]. However, the response time between $\mathrm{AC}$ and DC fields does not match the outcome of other experiments that recorded a slower response time in AC fields [16]. This discrepancy could be 
attributed to the use of higher-viscosity fluids, as the movement of particles in an AC field seems to be severely inhibited by a high viscosity in the base liquid $[13,16]$. In a silicone oil with a base liquid of 1000 centistokes $(\mathrm{cSt})$ viscosity, there was no $\mathrm{AC}$ response. It can be suggested that the response times usually reported for ER fluids of the order of a millisecond represent the time it takes for the first fibrils to start forming in the fluid. The size of the response is also greater in laboratory-prepared fluids, despite a slightly slower response time, when compared to commercial ER fluids [13].

Although the aforementioned studies explore some fundamental properties of ER fluid, the application of ER fluid outside the automobile industry remains very limited [17, 18]. Exploring the application of this technique to medical technologies could lead to exciting new gripping methodologies. A gravity-assisted gripping concept is explored in this paper. It relies on gravity to position the "fingers" (movable plates), which are then held in place through activation of the ER fluid. For this research, a single "finger" unit is tested. The aim of this study is to explore if a conceptual prototype of an electrorheological (ER) system could hold a simple geometrical shape when it is activated and subsequently quantify the force it could withstand (captured by the coefficient $\mu$ ). Different amounts of silica particles were dispersed in two silicone oils (10 cSt and $1000 \mathrm{cSt}$ ) to provide further information regarding the performance differences of diverse ER fluids.

\section{Materials and methods}

\section{Test rig}

A rig was built to contain the ER fluid, keep two plate electrodes $2 \mathrm{~mm}$ apart and parallel and provide movement of one plate along a vertical path. The design consisted of two Tslots (SolidWorks 2015, Dassault Systèmes, USA), and the rig was built out of unplasticized polyvinyl chloride (uPVC). The rig was bonded and sealed with uPVC glue (Fig. 2).

The design ensured that there was always an area of overlap throughout the measurement stage between the two electrodes of $25 \mathrm{~cm}^{2}$. This meant that $5 \mathrm{~mL}$ of fluid was activated during each experiment. The plate with the moveable electrode could be displaced during experimentation by a tensometer (Bose BioDynamic, USA). The tensometer was connected with the moveable part through a nylon PA66 cable to prevent electrical interference with the tensometer.

\section{Circuit}

A battery-powered system was designed to provide $8 \mathrm{kV}$ DC and generate a field strength of $4 \mathrm{kV} \mathrm{mm}^{-1}$ (Fig. 3a). This is in accordance with the strength that previously rec- ommended at which any effect would be marked [12]. The XP EMCO-C80 (a DC-HVDC converter) was used, which has the added benefit of a low-pass filter on the output. Extra safety measures included a double-switch system.

\section{ER fluid}

The ER fluids were chosen based on past work, suggesting that a sizeable response was achievable with a laboratory-prepared fluid [13]. Silica particles (Silica gel 60737, Sigma-Aldrich, USA) were to be dispersed in silicone oils (Sigma-Aldrich, USA) with a viscosity of $10 \mathrm{cSt}$ and $1000 \mathrm{cSt}$. Fluids with 10, 20, 30 and 40\% of silica particles by mass were prepared for both oils to give a range of conditions. The size of silica particle was chosen to maximize Brownian motion and prevent sedimentation as much as possible. While mixing the fluids, it was noted that the $40 \%$ compositions had completely adsorbed all the oil and resulted in an inhomogeneous mixture of saturated and dry particles that would be impossible to test. After the compositions had been mixed in the $10 \mathrm{cSt}$ oil, it was observed that substantial settling had occurred within 5 min of mixing. This meant that fluids with a $10 \mathrm{cSt}$ base liquid would need stirring between tensometer tests.

The fluids with $1000 \mathrm{cSt}$ base liquid viscosity did not have the issue of rapid settling out, and it took several hours before any appreciable settling of particles occurred. Mixing did cause air bubbles to become trapped in these fluids. The viscosity of the liquid then prevented the bubbles from escaping, particularly for the $30 \%$ of silica particles by mass. However, for the $10 \mathrm{cSt}$ and lower values of particle by mass the bubbles were rising out of the liquid within an hour.

\section{Silica particles}

The water content of the silica particles $\left(\mathrm{SiO}_{2}\right.$ gel $)$ can affect the formation of water bridges. To verify the exact water content, a verification experiment was run on a subset of the particles. These sample particles were heated for $75 \mathrm{~min}$ at $150{ }^{\circ} \mathrm{C}$ in a Memmert laboratory oven, and the mass difference was measured to calculate the water content. A total of 29 samples of approximately $1 \mathrm{~g}$ were placed in $10-\mathrm{mL}$ Pyrex beakers and positioned in the oven. A mean water content of $5.19 \%$ with a standard deviation of 0.57 was found across all the samples. The full range of water content was $3.88-6.42 \%$, which was within the $3-7 \%$ range that was mentioned by Sigma-Aldrich for these particles.

\section{Force measurements}

The tensometer was set under displacement control using a specific displacement profile (Fig. 3b). A miniature sealed stainless steel load cell (WMC-5, Interface Force Measure- 

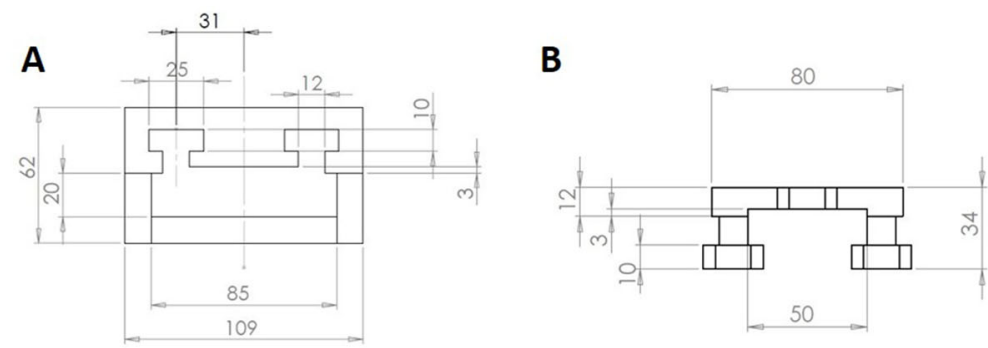

C
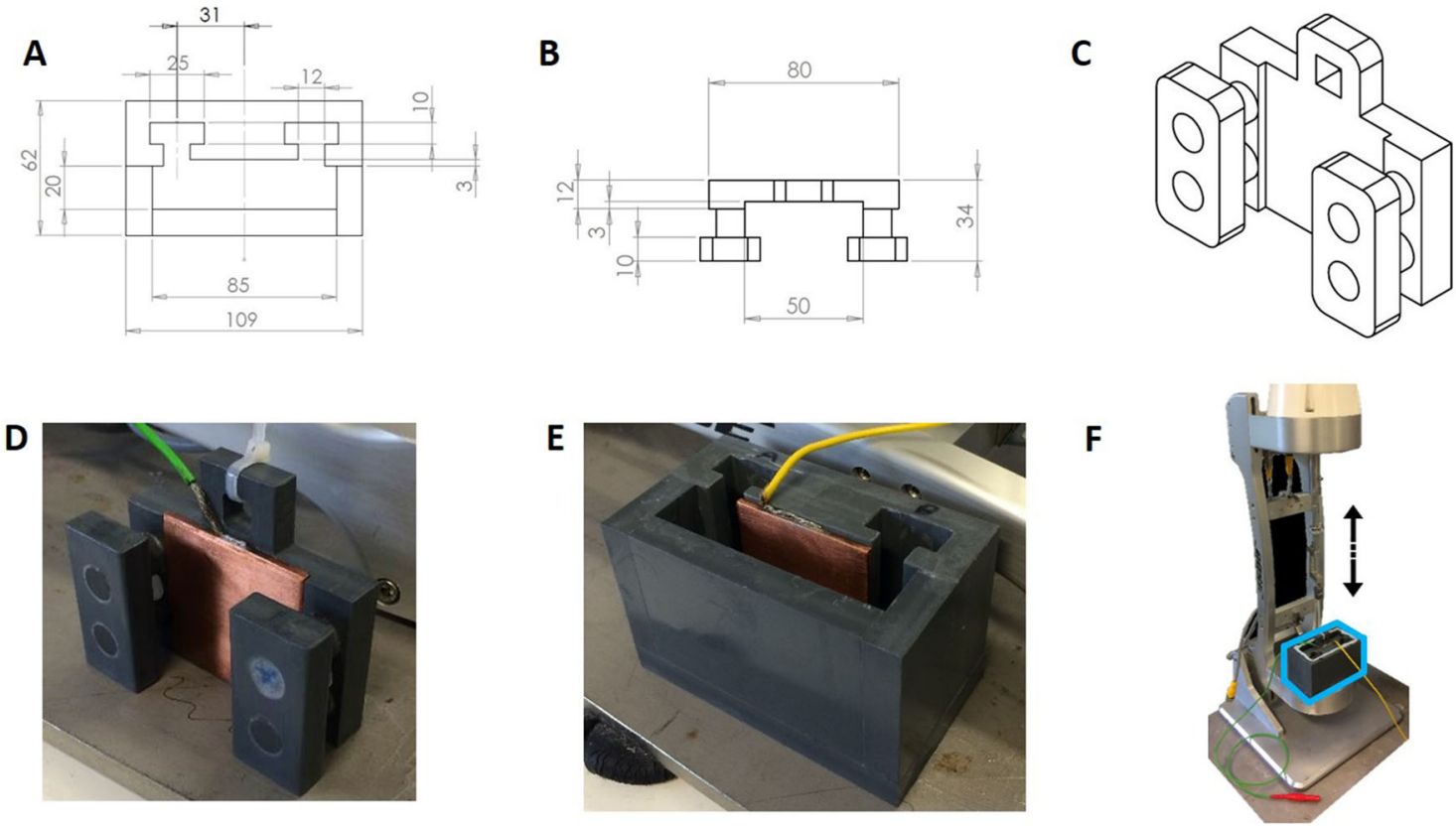

Fig. 2 Test rig used for proof-of-concept testing. a the top view of the fluid container with all dimensions given in $\mathrm{mm}$; $\mathbf{b}$ the top view of the movable plate with all dimensions given in $\mathrm{mm}$; a perspective view of the movable plate; $\mathbf{d}$ picture of the moveable plate with the electrode attached. The plate was made from unplasticized polyvinyl chloride
(uPVC); e picture of the container with the electrode attached. The container was also made from uPVC; $\mathbf{f}$ the experimental setup with the movable plate attached to the tensometer. The container with the movable plate is highlighted in blue. The arrows show the possible directions of displacement ments Ltd., UK) was used to obtain force measurements. This displacement profile provided a steady state of around $5 \mathrm{~s}$ during which the system was in equilibrium. The average force value was taken when the system was in equilibrium. A simple visualization of the forces is shown in Fig. 4.

During equilibrium, the following is true:

$F_{\mathrm{t}}=F_{\mathrm{g}}+F_{\mathrm{f}}+F_{\mathrm{ER}}$

$F_{\mathrm{g}}$ is the product of gravity acting upon the mass of the moveable part, $F_{\mathrm{f}}$ is the summed forces related to the fluid and object interaction, $F_{\mathrm{t}}$ is the force generated by the tensometer, and $F_{\mathrm{ER}}$ is the force that arises due to the activation of the fluid. The value for $F_{\mathrm{t}}$ is obtained by averaging the values from the tensometer during the steady-state period in which the system is in equilibrium. The following equation was then applied to determine the activation effect of the ER fluid:

$\mu=\frac{F_{i j}}{F_{\mathrm{B}}}-1$

$F_{\text {B }}$ represents the baseline measurement that consisted of the average $F_{\mathrm{t}}$ value for the best lubricated system (deactivated $10 \mathrm{cSt}$ oil with no particles). $F_{i j}$ is the $F_{\mathrm{t}}$ values for the silicone oils ( $i=1$ for $1000 \mathrm{cSt}$ and $i=2$ for $10 \mathrm{cSt}$ ) and particles $(j=1$ for $10 \% ; j=2$ for $20 \% ; j=3$ for $30 \%$; and $j=4$ for $40 \%$ of silica particles by mass). The best lubricated system will thus yield a $\mu$ value of zero $\left(\frac{F_{\mathrm{B}}}{F_{\mathrm{B}}}-1=0\right)$. In this case, only the force required to lift up the moveable part is measured by the tensometer. To show that the system can "hold" the moveable part by itself requires the activated fluid to have a $\mu$ of $1\left(\frac{2 F_{\mathrm{B}}}{F_{\mathrm{B}}}-1=1\right)$, which indicates that double the amount of force should be measured. Anything above a $\mu$ of 1 implies that the system can "hold" more than just the movable part alone. In other words, the parameter $\mu$ will go to zero if the activation does not have any effect and $\mu \geq 1$ arises if the system can "hold" its shape/position. The parameter $\mu$ provides a single value that represents the capabilities of the system to hold a simple geometric shape under a specific condition.

Each of the aforementioned mixtures of silicone oils and particles was tested 10 times, both with activation and without activation. The order of testing was randomized to prevent any sequence-induced effects.

\section{Statistical analysis}

The data followed a Gaussian distribution, when visualized using histograms and tested with a one-sample Kolmogorov-Smirnov. An analysis of variance (ANOVA) test was performed between each of the aforementioned mixtures of silicone oils and particles during both activation and 


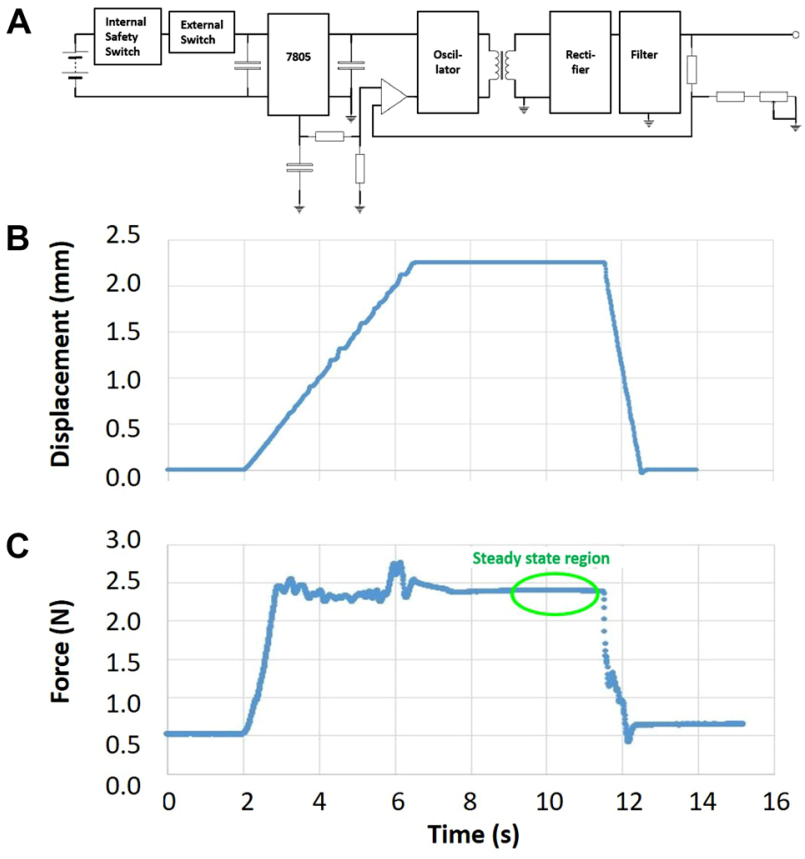

Fig. 3 a The circuit design for the battery-powered ER fluid system. b Displacement profile of the tensometer used during testing. c Typical force-time graph produced during testing in which the steady-state region (circled in green) represents the equilibrium. All time is in seconds (s), displacement is in millimeters ( $\mathrm{mm}$ ) and force is in Newton (N)

Fig. 4 A basic free-body diagram of the movable plate that sits in the ER fluid and is attached to the tensometer. $F_{\mathrm{g}}$ is the product of the mass (in $\mathrm{Kg}$ ) of the moveable part and the constant of gravitation acceleration (in $\mathrm{m} / \mathrm{s}^{2}$ ), $F_{\mathrm{f}}$ is the summed forces related to the fluid and object interaction, $F_{\mathrm{t}}$ is the force generated by the tensometer, and $F_{\mathrm{ER}}$ is the force that arises due to the activation of the fluid

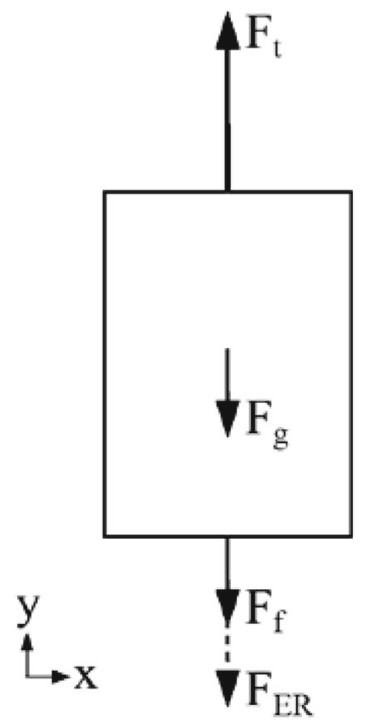

deactivation. An outcome bias could occur due to the relative small number of samples that were analyzed with the Kolmogorov-Smirnov test. However, the ANOVA has been shown to be robust to violations of the normality assumption [19]. A Holm-Bonferroni method was applied to find significant differences between activation and deactivation within the conditions. The Holm-Bonferroni method is designed to counteract the increase in Type I error that occurs when sev- eral hypotheses are considered simultaneously and therefore corrects for the multiple tests that were run [20].

All data and statistical analysis were completed using MATLAB (R2014b, MathWorks, USA) and Excel (Office 2016, Microsoft, USA).

\section{Results}

A scatter plot of the parameter $\mu$ is provided in Fig. 5, showing the different fluid compositions during activated and deactivated states. The system mechanically jammed on two occasions due to misalignment, and this has been highlighted in the figure. These points were excluded from the subsequent analysis.

Significant differences were found among the conditions $(F(14,135)=19.80, p<0.001)$, and the post hoc test revealed that the largest significant difference (mean \pm standard deviation), between activation and deactivation, was found for the $10 \mathrm{cSt}$ silicone oil with $30 \%$ silica particles condition $\left(\mu_{\text {deactivation }}=0.58 \pm 0.17\right.$ vs. $\mu_{\text {active }}=1.04 \pm 0.25, p$ $<0.001)$. Activating the $10 \mathrm{cSt}$ silicone oil with $30 \%$ silica particles created the only condition during which the average $\mu \geq 1$. No difference was found in the measured force when the silica particles were removed from the oil $\left(\mu_{\text {deactivation }}=0.01 \pm 0.01\right.$ vs. $\mu_{\text {active }}=-0.01 \pm 0.01, \mathrm{p}=$ N.S.). The greatest significant difference in means among the $1000 \mathrm{cSt}$ oils appeared during the $10 \%$ silica particles con-

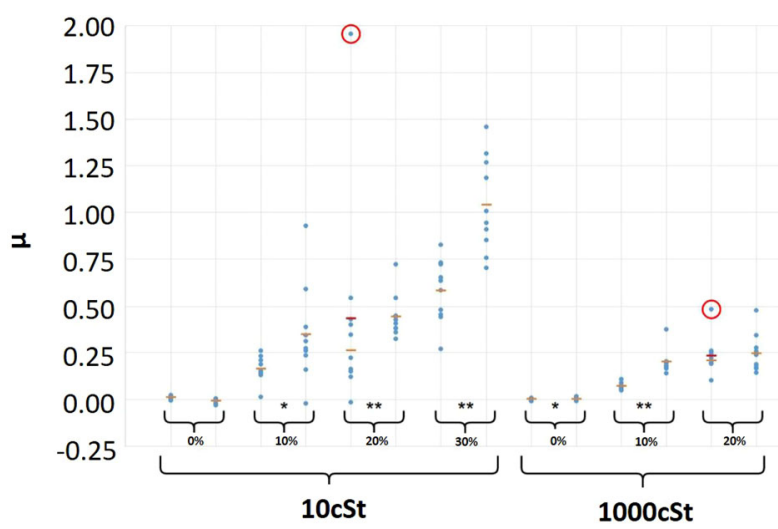

Fig. 5 A scatter plot of the parameter $\mu$ for the different fluid compositions during deactivated (left of each pair) and activated (right of each pair) states. Data points during which mechanical jamming took place are highlighted by red circles. Average values for each condition are denoted by horizontal red lines. Orange lines show the average values when the data points during which jamming occurred were excluded. The viscosity of the $1000 \mathrm{cSt}$ silicone oil with $30 \%$ (and higher) silica particles entrapped air bubbles that could not be released, which made it impossible to obtain suitable data for this condition. The $40 \%$ compositions resulted in an inhomogeneous mixture of saturated and dry particles and were therefore not testable. A single asterisk $(*)$ indicates that a $p$ value $<0.01$ in post hoc testing was found for that specific pair. A double asterisk (**) indicates a $p$ value $<0.001$ 
dition $\left(\mu_{\text {deactivation }}=0.07 \pm 0.02\right.$ vs. $\mu_{\text {active }}=0.20 \pm 0.06$, $p<0.001)$.

\section{Discussion}

The aim of this study was to explore if a conceptual prototype of an electrorheological (ER) system could hold a geometric shape when it is activated (as indicated by a $\mu \geq 1$ ). It was found that during the activation of a $10 \mathrm{cSt}$ silicone oil, with $30 \%$ silica particles, the position of the system could be maintained purely by the activation of the ER fluid. This was the only condition during which $\mu$ was greater than unity. It showed that the activated fluid was capable of supporting more than just its own weight and behaved more as a solid than a liquid. As the determination of $\mu$ was based on liquid behavior assumptions, it is of limited validity when the value of $\mu$ goes beyond unity. However, it is still representative of the response's magnitude. The difference between $\mu$ observed between the activated and deactivated states is preferably as large as possible, as a large difference would indicate that the states truly differ. The largest difference between the average $\mu$ values was also found for the $10 \mathrm{cSt}$ silicone oil, with $30 \%$ silica particles condition.

The difference between liquid viscosities will have had an effect on wettability. Wettability of the oil is controlled by the balance between the liquid-to-liquid and the liquidto-surface intermolecular interactions [21]. It is the ability of the oil to maintain contact with a solid surface (e.g., particles). The wettability decreases with higher oil viscosities, which indicates larger yield stresses for oils with a lower viscosity [22]. This might explain some of the observed effects between the conditions.

The equilibrium was used to obtain an average force value from the tensometer. The variation in force readings was always less than $0.1 \mathrm{~N}$ during the steady state, and the vast majority varied less than $0.03 \mathrm{~N}$. This suggests that any selected steady-state force would have yielded very similar results.

The assumption that activation should have no effect on the pure oils was supported by the results of this study. The 10 cSt with $0 \%$ silica particles had similar averages between the activated and deactivated states $\left(\mu_{\text {deactivation }}=0.01 \pm 0.01\right.$ vs. $\mu_{\text {active }}=-0.01 \pm 0.01, p=$ N.S.), and the same was the case with the $1000 \mathrm{cSt}$ with $0 \%$ silica particles $\left(\mu_{\text {deactivation }}\right.$ $=0.00 \pm 0.01$ vs. $\mu_{\text {active }}=0.00 \pm 0.01, p=$ N.S. $)$.

The $1000 \mathrm{cSt} 10 \%$ composition was the least useful composition tested. As the composition is very viscous, bubbles got incorporated into the fluid during mixing and it took a long time for them to rise out. Nevertheless, another study previously observed that the torque can be increased by a factor of 1.5 when $1000 \mathrm{cSt}$ fluids are used instead of $100 \mathrm{cSt}$ fluids [13]. It could therefore be expected that despite the dif- ferent measurement approaches of that study and this paper, a higher $\mu$ might be found for the $1000 \mathrm{cSt}$ fluid instead of the $10 \mathrm{cSt}$ oil. However, the results in Fig. 5 show an opposite trend. The difference between these two studies could be explained by the fact that [13] used a horizontal and completely submerged test setup. Their experimental design provides a submersion of the electrodes in the ER fluid throughout the test cycle, and the outcomes might be a property of a specific system. This suggests the need to keep testing future iterations of the design in order to ascertain validity of a changing ER fluid gripping system. The difference between the two studies might further be explained by the variation of other important variables, such as the electric field $\left(4 \mathrm{kV} \mathrm{mm}^{-1}\right.$ vs. $\left.2 \mathrm{kV} \mathrm{mm}^{-1}\right)$, that was used. In general, there are five primary factors that need to be taken into account for a given solid-liquid pairing to be able to maximize the formation of water bridges and thus the size of the response. They consist of particle concentration, base liquid viscosity, water content of the solid particles, field strength and surface area of the electrodes. This paper varied particle concentration and base liquid viscosity and kept all other factors constant. All these factors can be optimized further to provide the best possible functionality for an ER-enabled gripper.

The use of smart materials for actuation still faces many material and technical limitations before a practical implementation is possible [23]. However, there are already developments taking place to use these fluids for the creation of machines, such as lower limb prosthesis, to reduce unwanted vibrations in real time [23]. As mentioned earlier, ER fluid joints and pistons have also been explored for minimally invasive surgery [10]. The joints consist of a ball (inner electrode) located in a spherical housing (outer electrode) with the ER fluid between them. This could potentially be adapted to represent the interphalangeal joints, which consist of simple hinge joints. This approach would yield a more anatomically correct representation of the gripper, but it comes at a greater control complexity of the system. Position control can also be accomplished by using ER clutch actuators [24]. Yet, a gravity-assisted method would even further increase the mechanical simplicity. Nonetheless, the gravityassisted approach does require a specific directionality of movement, as gravity is required to position the "fingers." It has been shown that a "finger" (movable plate) can hold its position against gravity, but the design needs to be further optimized in order to hold objects of greater mass. The number of movable units can be increased to fulfill this design criteria. A gravity-based ER fluid gripper with five "fingers" was virtually prototyped to hold complex shapes (see supplementary video S1). The five "fingers" were embedded in a container that allowed for a simple translational movement to occur. The ER fluid was activated when all "fingers" were 
in contact, which then allowed for the low-mass object to be moved during this locked stage.

For any future experiments, it is recommended that the device uses nonstick smooth surfaces with the electrodes embedded, as this would further reduce the occurrence of jamming. To minimize other mechanical issues, it is also suggested that there is space left below the electrode so that settled particles do not tilt the moving part out of alignment and again increase the risk of jamming. The prototype can now be extended to include multiple movable plates to create more complex geometrical shapes. A sealed top will keep the fluid in the container to allow for further practical testing of the system (as suggested in supplementary video S1). Key design criteria will be the insulation of the system in order to create a safe device for everyday use.

The most basic and often used gripper in prosthetics is a hook. The split-hook design consists of the two hook-shaped steel fingers. More complex advanced multi-fingered prosthetics also come with more degrees of freedom that need to be controlled. A great benefit of a gravity-assisted fluidbased system is that it has a simple control mechanism, with the "fingers" moving around an object and then locking in that geometrical position during activation. The binary control provides simplicity similar to the hook design, while the multitude of fingers of the smart fluid system allows for complex grasp types to be attained, which are not possible with the hook. The fluid-based system aims to combine the best of both worlds. The prototype unit now needs to be duplicated and scaled to create a more suitable gripping configuration. The initial results are very promising, but the usability of the system depends greatly on satisfying many other more practical user requirements. The development of such a device for practical purposes should follow a user-centered design approach to ensure that the gripping system is fit for human purposes. The inclusion of preferences of patients and clinicians is needed to create a technology that is applicable in the real world [25].

Finally, cellulose particles and corn starch have been used for ER fluid before and provide biodegradable materials that are abundant in nature [26]. This provides the ability to develop these systems in a way that reduces device corrosion, but allow for true integration of systems and create new bionic technologies. This work opens the door to explore new designs and manufacturing approaches for assistive technologies.

A proof-of-concept ER fluid-based system has been tested, and it has been shown that the system can support an increased force when activated. The outcome indicates that an ER fluid composition exists that can reliably support more than the weight of the system and hold a simple geometrical shape. The promising nature of a smart (ER) fluid-based system could provide new ways in the future to develop systems that can help users grasp objects and thus better support everyday living activities of patients in need of such technologies.

Acknowledgements The authors like to acknowledge Jiaju Du for supporting the electrical design and Cleveland Williams \& Maurice Keeble-Smith for supporting the mechanical design.

Author Contributions Jeroen Bergmann contributed to the conceptualization, funding acquisition, project administration and supervision. Ivo Radice contributed to the data curation. Both Ivo Radice and Jeroen Bergmann contributed to the formal analysis, investigation, methodology, validation, visualization, writing - original draft, and writing—review and editing.

Funding This work was supported by the EPSRC Impact Acceleration Account Award [EP/R511742/1].

\section{Compliance with ethical standard}

Conflicts of interest The authors declare no conflict of interest. The funders had no role in the design of the study; in the collection, analyses or interpretation of data; in the writing of the manuscript; or in the decision to publish the results.

Ethical approval No humans, animals or cells were used in the study.

Open Access This article is distributed under the terms of the Creative Commons Attribution 4.0 International License (http://creativecomm ons.org/licenses/by/4.0/), which permits unrestricted use, distribution, and reproduction in any medium, provided you give appropriate credit to the original author(s) and the source, provide a link to the Creative Commons license, and indicate if changes were made.

\section{References}

1. Bergmann JHM, Langdon PM, Mayagoitia RE, Howard N (2014) Exploring the use of sensors to measure behavioral interactions: an experimental evaluation of using hand trajectories. PLoS ONE 9:e88080

2. Yap HK, Lim JH, Nasrallah F, Goh JCH, Yeow RCH (2015) A soft exoskeleton for hand assistive and rehabilitation application using pneumatic actuators with variable stiffness. In: Proceedings of the 2015 IEEE international conference on robotics and automation (ICRA). IEEE, pp 4967-4972

3. Belter JT, Segil JL, Dollar AM, Weir RF (2013) Mechanical design and performance specifications of anthropomorphic prosthetic hands: a review. J Rehabil Res Dev 50:599-618

4. Xu Z, Todorov E (2016) Design of a highly biomimetic anthropomorphic robotic hand towards artificial limb regeneration. In: Proceedings of the 2016 IEEE international conference on robotics and automation (ICRA). IEEE, pp 3485-3492

5. Wang W, Ahn SH (2017) Shape memory alloy-based soft gripper with variable stiffness for compliant and effective grasping. Soft Robot 4(4):379-389

6. Brown E, Rodenberg N, Amend J, Mozeika A, Steltz E, Zakin MR, Lipson H, Jaeger HM (2010) Universal robotic gripper based on the jamming of granular material. Proc Natl Acad Sci 107:18809-18814

7. Eshaghi M, Sedaghati R, Rakheja S (2016) Dynamic characteristics and control of magnetorheological/electrorheological sandwich structures: a state-of-the-art review. J Intell Mater Syst Struct 27:2003-2037 
8. Madeja J, Kesy Z, Kesy A (2011) Application of electrorheological fluid in a hydrodynamic clutch. Smart Mater Struct 20:105005

9. Lu Q, Han W, Choi H, Lu Q, Han WJ, Choi HJ (2018) Smart and functional conducting polymers: application to electrorheological fluids. Molecules 23:2854

10. Oh J-S, Han Y-M, Lee S-R, Choi S-B (2013) A 4-DOF haptic master using ER fluid for minimally invasive surgery system application. Smart Mater Struct 22:045004

11. Nguyen QH, Choi SB, Lee YS, Han MS (2013) Optimal design of a new 3D haptic gripper for telemanipulation, featuring magnetorheological fluid brakes. Smart Mater Struct 22:015009

12. Stangroom JE (1983) Electrorheological fluids. Phys Technol $14: 290-296$

13. Nava R, Ponce MA, Rejón L, Víquez S, Castaño VM (1997) Response time and viscosity of electrorheological fluids. Smart Mater Struct 6:67-75

14. Winslow WM (1947) Undefined Method and means for translating electrical impulses into mechanical force. Google Patents. US Patent 2, 417,850

15. Conrad H, Sprecher AF (1991) Characteristics and mechanisms of electrorheological fluids. J Stat Phys 64:1073-1091

16. Shiang AH, Coulter JP (1996) A comparative study of AC and DC electrorheological material based adaptive structures in small amplitude vibration. J Intell Mater Syst Struct 7:455-469

17. Choi SB, Seong MS, Kim KS (2009) Vibration control of an electrorheological fluid-based suspension system with an energy regenerative mechanism. Proc Inst Mech Eng Part D J Automob Eng 223:459-469
18. Hartsock DL, Novak RF, Chaundy GJ (1991) ER fluid requirements for automotive devices. J Rheol 35:1305-1326

19. Harwell MR, Rubinstein EN, Hayes WS, Olds CC (1992) Summarizing Monte Carlo results in methodological research: the oneand two-factor fixed effects ANOVA cases. J Educ Stat 17:315-339

20. Hilm S (1979) A simple sequentially rejective multiple test procedure. JSTOR

21. Moldoveanu SC, David V (2017) RP-HPLC analytical columns. In: Selection of the HPLC method in chemical analysis. Elsevier, pp 279-328. https://doi.org/10.1016/b978-0-12-803684-6.00007-

22. Ma N, Dong X (2017) Effect of carrier liquid on electrorheological performance and stability of oxalate group-modified $\mathrm{TiO}_{2}$ suspensions. J Wuhan Univ Technol Mater Sci Ed 32(4):854-861

23. Sohn JW, Kim G-W, Choi S-B (2018) A state-of-the-art review on robots and medical devices using smart fluids and shape memory alloys. Appl Sci 8(10):1928

24. Han S-S, Choi S-B, Cheong C-C (2000) Position control of X-Y table mechanism using electro-rheological clutches. Mech Mach Theory 35:1563-1577

25. Bergmann J, Chandaria V, McGregor A (2012) Wearable and implantable sensors: the patient's perspective. Sensors 12(12):16695-16709

26. Choi K, Gao C, Nam J, Choi H (2017) Cellulose-based smart fluids under applied electric fields. Materials 10(9):1060 